\title{
ETA-RICCI SOLITONS ON LP-SASAKIAN MANIFOLDS
}

\author{
PRADIP MAJHI AND DEBABRATA KAR
}

\begin{abstract}
We consider $\eta$-Ricci solitons on Lorentzian para-Sasakian manifolds with Codazzi type of the Ricci tensor. Then we study $\eta$-Ricci solitons on $\varphi$-conformally semi-symmetric, $\varphi$-Ricci symmetric, and conformally Ricci semi-symmetric Lorentzian para-Sasakian manifolds. Finally, we construct an example of a three dimensional Lorentzian para-Sasakian manifold which admits $\eta$-Ricci solitons with non-constant scalar curvature.
\end{abstract}

\section{INTRODUCTION}

In 1982, R. S. Hamilton [18 introduced the notion of Ricci flow to find a canonical metric on a smooth manifold. The Ricci flow is an evolution equation for metrics on a Riemannian manifold $M$ defined as follows:

$$
\frac{\partial}{\partial t} g=-2 S
$$

where $S$ denotes the Ricci tensor. Ricci solitons are special solutions of the Ricci flow equation (1.1) of the form $g=\sigma(t) \psi_{t}^{*} g$ with the initial condition $g(0)=g$, where $\psi_{t}$ are diffeomorphisms of $M$ and $\sigma(t)$ is the scaling function. A Ricci soliton is a generalization of an Einstein metric. We recall the notion of Ricci solitons according to [6]. On the manifold $M$, a Ricci soliton is a triple $(g, V, \lambda)$ with $g$ a Riemannian metric, $V$ a vector field (called the potential vector field), and $\lambda$ a real scalar such that

$$
\mathcal{L}_{\xi} g+2 S+2 \lambda g=0
$$

where $\mathcal{L}$ is the Lie derivative. Metrics satisfying $(1.2)$ are interesting and useful in physics and are often referred to as quasi-Einstein metrics ([8, 9]). Compact Ricci solitons are the fixed points of the Ricci flow $\frac{\partial}{\partial t} g=-2 S$, projected from the space of metrics onto its quotient modulo diffeomorphisms and scalings, and often arise blow-up limits for the Ricci flow on compact manifolds. Theoretical physicists have also been looking into the equation of Ricci solitons in relation with string theory. The initial contribution in this direction is due to Friedan [16], who discusses some of its aspects.

2010 Mathematics Subject Classification. 53C15, 53C25.

Key words and phrases. Ricci solitons; $\eta$-Ricci solitons; Lorentzian para-Sasakian manifolds; Codazzi type of the Ricci tensor.

Debabrata Kar is supported by the Council of Scientific and Industrial Research, India (File no. 09/028(1007)/2017-EMR-1). 
Ricci solitons have been studied by many authors, such as [14, 15, 18, 19] and several others.

As a generalization of Ricci solitons, the notion of $\eta$-Ricci solitons was introduced by Cho and Kimura [11]. This notion has also been studied in [6], for Hopf hypersurfaces in complex space forms. An $\eta$-Ricci soliton is a tuple $(g, V, \lambda, \mu)$, where $V$ is a vector field on $M, \lambda$ and $\mu$ are real constants, and $g$ is a Riemannian (or pseudo-Riemannian) metric satisfying the equation

$$
\mathcal{L}_{\xi} g+2 S+2 \lambda g+2 \mu \eta \otimes \eta=0 .
$$

In this connection we mention the works of Blaga [3, 2] and Prakasha et al. [25] on $\eta$-Ricci solitons. In particular, if $\mu=0$, then the notion of $\eta$-Ricci solitons $(g, V, \lambda, \mu)$ reduces to the notion of Ricci solitons $(g, V, \lambda)$. If $\mu \neq 0$, then the $\eta$ Ricci solitons are called proper $\eta$-Ricci solitons. We refer to [1, 5, 23, and references therein for a survey and further references on the geometry of Ricci solitons on pseudo-Riemannian manifolds.

Gray [17] introduced the notion of Codazzi type of the Ricci tensor. A pseudoRiemannian manifold is said to satisfy Codazzi type of the Ricci tensor if its Ricci tensor $S$ of type $(0,2)$ is non-zero and satisfies the condition

$$
\left(\nabla_{X} S\right)(Y, Z)=\left(\nabla_{Y} S\right)(X, Z)
$$

which implies that $\operatorname{div} R=0$, where div denotes divergence and $R$ is the Riemannian curvature tensor of type $(1,3)$.

A Riemannian or pseudo-Riemannian manifold $(M, g)$ is called locally symmetric [7] if $\nabla R=0$, where $R$ is the manifold's Riemannian curvature tensor. A Riemannian or pseudo-Riemannian manifold $(M, g), n \geq 3$, is said to be semi-symmetric if the curvature condition

$$
R \cdot R=0
$$

holds, where $R$ denotes the curvature tensor of the manifold. It is well known that the class of semi-symmetric manifolds includes the set of locally symmetric manifolds $(\nabla R=0)$ as a proper subset. Semi-symmetric Riemannian manifolds were firstly studied by E. Cartan, A. Lichnerowich, R. S. Couty, and N. S. Sinjukov. A fundamental study on Riemannian semi-symmetric manifolds was introduced by Z. I. Szabó [26]. Later E. Boeckx et al. [4] and O. Kowalski 20] and many others have studied semi-symmetric manifolds.

In [29] Yildiz et al. studied $\varphi$-conformally semi-symmetric $(k, \mu)$-contact manifolds. A contact metric manifold is said to be $\varphi$-conformally semi-symmetric if $C \cdot \varphi=0$, where $C$ is the conformal curvature tensor. Moreover, conformally Ricci semi-symmetric manifolds, that is, $C \cdot S=0$, have been studied by Verstraelen [28. Motivated by the above studies, in the present paper we consider $\eta$-Ricci solitons on Lorentzian para-Sasakian manifolds with the curvature conditions $C \cdot \varphi=0$ and $C \cdot S=0$.

The study of curvature properties is one of the main problems in differential geometry. As S. S. Chern said in [10, "a fundamental notion is curvature in its different forms". Therefore, the determination of the Riemann curvature tensor constitutes a very important topic. In this sense, in the present paper we characterize 
$\eta$-Ricci solitons on LP-Sasakian manifolds satisfying certain curvature conditions. The paper is organized as follows:

After preliminaries in Section 3, we study $\eta$-Ricci solitons on Lorentzian paraSasakian manifolds (in short, LP-Sasakian manifolds). Section 4 is devoted to study $\eta$-Ricci solitons on LP-Sasakian manifolds satisfying Codazzi type of the Ricci tensor. In the next section, we study $\eta$-Ricci solitons on $\varphi$-conformally semisymmetric LP-Sasakian manifolds. In Section 6 , we consider $\eta$-Ricci solitons on $\varphi$-Ricci symmetric LP-Sasakian manifolds. Section 7 deals with the study of $\eta$-Ricci solitons on conformally Ricci semi-symmetric LP-Sasakian manifolds. Finally, we construct an example of a three dimensional LP-Sasakian manifold which admits $\eta$-Ricci solitons with non-constant scalar curvature.

\section{LP-SASAKIAN MANIFOLDS}

In 1989, Matsumoto [21] introduced the notion of Lorentzian para-Sasakian manifolds or, in short, LP-Sasakian manifolds. An example of a five dimensional LP-Sasakian manifold was given by Matsumoto, Mihai and Rosaca [22].

Let $M$ be an $n$-dimensional differential manifold endowed with a $(1,1)$ tensor field $\varphi$, a vector field $\xi$, a 1 -form $\eta$, and a Lorentzian metric $g$ of type $(0,2)$ such that for each point $p \in M$, the tensor $g_{p}: T_{p} M \times T_{p} M \rightarrow \mathbb{R}$ is a non-degenerate inner product of signature $(-,+,+, \ldots,+)$, where $T_{p} M$ denotes the tangent space of $M$ at $p$ and $\mathbb{R}$ is the real number space which satisfies

$$
\begin{gathered}
\varphi^{2}(X)=X+\eta(X) \xi, \eta(\xi)=-1, \\
g(X, \xi)=\eta(X), \quad g(\varphi X, \varphi Y)=g(X, Y)+\eta(X) \eta(Y),
\end{gathered}
$$

for all vector fields $X, Y$. Then, such a structure $(\varphi, \xi, \eta, g)$ is termed as Lorentzian almost paracontact structure and the manifold with the structure $(\varphi, \xi, \eta, g)$ is called a Lorentzian almost paracontact manifold. In the Lorentzian almost paracontact manifold $M$, the following relations hold [21]:

$$
\begin{gathered}
\varphi \xi=0, \quad \eta(\varphi X)=0, \\
\Phi(X, Y)=\Phi(Y, X),
\end{gathered}
$$

where $\Phi(X, Y)=g(X, \varphi Y)$.

A Lorentzian almost paracontact manifold $M$ equipped with the structure $(\varphi, \xi$, $\eta, g)$ is called an LP-Sasakian manifold if

$$
\left(\nabla_{X} \varphi\right) Y=g(\varphi X, \varphi Y) \xi+\eta(Y) \varphi^{2} X
$$

where $\nabla$ denotes the operator of covariant differentiation with respect to the Lorentzian metric $g$. In an LP-Sasakian manifold $M$ with the structure $(\varphi, \xi, \eta, g)$, it is easily seen that

$$
\begin{gathered}
\nabla_{X} \xi=\varphi X \\
\left(\nabla_{X} \eta\right)(Y)=g(\varphi X, Y)=\left(\nabla_{Y} \eta\right)(X) \\
R(\xi, X) Y=g(X, Y) \xi-\eta(Y) X \\
R(X, Y) \xi=\eta(Y) X-\eta(X) Y, \\
S(X, \xi)=(n-1) \eta(X),
\end{gathered}
$$


for all vector fields $X, Y$ on $M$. LP-Sasakian manifolds have been studied by several authors such as [12, 24, 27] and many others.

Notice that the Ricci tensor $S$ and the scalar curvature $r$ are defined by

$$
S(X, Y)=\sum_{i=1}^{n} \epsilon_{i} g\left(R\left(e_{i}, X\right) Y, e_{i}\right)
$$

and

$$
r=\sum_{i=1}^{n} \epsilon_{i} S\left(e_{i}, e_{i}\right),
$$

where $\left\{e_{i}\right\}$ is an orthonormal basis such that $e_{1}=\xi$ and we put $\epsilon_{i}=g\left(e_{i}, e_{i}\right)$, that is, $\epsilon_{1}=-1, \epsilon_{2}=1, \ldots, \epsilon_{n}=1$.

The conformal curvature tensor $C$ is defined by

$$
\begin{aligned}
C(X, Y) Z= & R(X, Y) Z-\frac{1}{n-2}[S(Y, Z) X-S(X, Z) Y \\
& +g(Y, Z) Q X-g(X, Z) Q Y] \\
& +\frac{r}{(n-1)(n-2)}[g(Y, Z) X-g(X, Z) Y],
\end{aligned}
$$

where $S$ is the Ricci tensor, $Q$ is the Ricci operator defined by $S(X, Y)=g(Q X, Y)$, and $r$ is the scalar curvature of the manifold $M$.

Definition 2.1. A pseudo-Riemannian manifold $M$ of dimension $n$ is said to be an $\eta$-Einstein manifold if the Ricci tensor $S$ of $M$ satisfies the relation

$$
S(X, Y)=a g(X, Y)+b \eta(X) \eta(Y),
$$

where $a, b$ are smooth functions.

Definition 2.2. If $(M, V, \lambda, \mu)$ is an $\eta$-Ricci soliton, then the 1 -form $\xi$ is said to be a potential vector field.

For $\eta$-Ricci solitons on LP-Sasakian manifolds, we observe the following.

Proposition 2.3. For an $\eta$-Ricci soliton on an LP-Sasakian manifold, the Ricci tensor $S$ is of the form

$$
S(X, Y)=-g(\varphi X, Y)-\lambda g(X, Y)-\mu \eta(X) \eta(Y)
$$

and

$$
-\lambda+\mu=n-1 \text {. }
$$

Remark 2.4. The above form of the Ricci tensor is also deduced by Blaga in [3, p. 492].

Example 2.5. We consider the 3-dimensional manifold $M=\left\{(x, y, z) \in \mathbb{R}^{3}\right\}$, where $(x, y, z)$ are the standard coordinates of $\mathbb{R}^{3}$.

The vector fields

$$
E_{1}=e^{z} \frac{\partial}{\partial y}, \quad E_{2}=e^{z}\left(\frac{\partial}{\partial x}+\frac{\partial}{\partial y}\right), \quad E_{3}=\frac{\partial}{\partial z}
$$

are linearly independent at each point of $M$. 
Let $g$ be the Lorentzian metric defined by

$$
\begin{gathered}
g\left(E_{1}, E_{1}\right)=g\left(E_{2}, E_{2}\right)=1, \quad g\left(E_{3}, E_{3}\right)=-1, \\
g\left(E_{1}, E_{2}\right)=g\left(E_{1}, E_{3}\right)=g\left(E_{2}, E_{3}\right)=0 .
\end{gathered}
$$

Let $\eta$ be the 1 -form defined by $\eta(Z)=g\left(Z, E_{3}\right)$ for any vector field $Z \in \chi(M)$. Let $\phi$ be the $(1,1)$ tensor field defined by

$$
\phi\left(E_{1}\right)=-E_{1}, \quad \phi\left(E_{2}\right)=-E_{2}, \quad \phi\left(E_{3}\right)=0 .
$$

Then using the linearity of $\phi$ and $g$ we have

$$
\begin{gathered}
\eta\left(E_{3}\right)=-1, \\
\phi^{2} Z=Z+\eta(Z) E_{3}, \\
g(\phi Z, \phi W)=g(Z, W)+\eta(Z) \eta(W),
\end{gathered}
$$

for any vector fields $Z, W \in \chi(M)$.

Then for $E_{3}=\xi$, the structure $(\phi, \xi, \eta, g)$ defines a Lorentzian paracontact structure on $M$.

Let $\nabla$ be the Levi-Civita connection with respect to the Lorentzian metric $g$ and $R$ be the curvature tensor of $g$. Then we have

$$
\left[E_{1}, E_{2}\right]=0, \quad\left[E_{1}, E_{3}\right]=-E_{1}, \quad\left[E_{2}, E_{3}\right]=-E_{2} .
$$

Taking $E_{3}=\xi$ and using Koszul's formula for the Lorentzian metric $g$, we can easily calculate

$$
\begin{array}{lll}
\nabla_{E_{1}} E_{1}=-E_{3}, & \nabla_{E_{1}} E_{2}=0, & \nabla_{E_{1}} E_{3}=-E_{1}, \\
\nabla_{E_{2}} E_{1}=0, & \nabla_{E_{2}} E_{2}=-E_{3}, & \nabla_{E_{2}} E_{3}=-E_{2}, \\
\nabla_{E_{3}} E_{1}=0, & \nabla_{E_{3}} E_{2}=0, & \nabla_{E_{3}} E_{3}=0 .
\end{array}
$$

From the above it can be easily seen that $M^{3}(\phi, \xi, \eta, g)$ is an LP-Sasakian manifold. With the help of the above results we can easily obtain

$$
\begin{array}{lll}
R\left(E_{1}, E_{2}\right) E_{3}=0, & R\left(E_{2}, E_{3}\right) E_{3}=-E_{2}, & R\left(E_{1}, E_{3}\right) E_{3}=-E_{1}, \\
R\left(E_{1}, E_{2}\right) E_{2}=E_{1}, & R\left(E_{2}, E_{3}\right) E_{2}=-E_{3}, & R\left(E_{1}, E_{3}\right) E_{2}=0, \\
R\left(E_{1}, E_{2}\right) E_{1}=-E_{2}, & R\left(E_{2}, E_{3}\right) E_{1}=0, & R\left(E_{1}, E_{3}\right) E_{1}=-E_{3} .
\end{array}
$$

From the above expressions the Ricci tensor is given by

$$
\begin{aligned}
S\left(E_{1}, E_{1}\right) & =g\left(R\left(E_{1}, E_{2}\right) E_{2}, E_{1}\right)-g\left(R\left(E_{1}, E_{3}\right) E_{3}, E_{1}\right) \\
& =2 .
\end{aligned}
$$

Similarly we have

$$
S\left(E_{2}, E_{2}\right)=2, \quad S\left(E_{3}, E_{3}\right)=-2
$$

and

$$
S\left(E_{i}, E_{j}\right)=0 \quad(i \neq j) .
$$

From (2.8) we obtain $S\left(E_{1}, E_{1}\right)=1-\lambda$ and $S\left(E_{3}, E_{3}\right)=\lambda-\mu$, therefore $\lambda=-1$ and $\mu=1$. The data $(g, \xi, \lambda, \mu)$ defines an $\eta$-Ricci soliton on the LP-Sasakian manifold. 
In view of the expressions of the Ricci tensor it is clear that the manifold is $\phi$-Ricci symmetric and $r=S\left(E_{1}, E_{1}\right)+S\left(E_{2}, E_{2}\right)-S\left(E_{3}, E_{3}\right)=6$.

In view of 2.10 we have

$$
Q E_{1}=2 E_{1}, \quad Q E_{2}=2 E_{2}, \quad Q E_{3}=2 E_{3} .
$$

From the above results it can be easily seen that $\varphi^{2}\left(\nabla_{X} Q\right) Y=0$ and $\psi=$ trace $\varphi=-2$. Using the values $n=3$ and $\psi=-2$, from Theorem 5.1 we get $\lambda=$ -1 and $\mu=1$, which are the same as we get in this example. Hence Theorem 5.1 is verified.

3. $\eta$-RICCI SOLITONS ON LP-SASAKIAN MANIFOLDS WITH CONSTANT SCALAR CURVATURE SATISFYing CODAZZI TYPE OF THE RiCCI TENSOR

Taking covariant differentiation of (2.8) with respect to $Z$ we get

$$
\begin{aligned}
\left(\nabla_{Z} S\right)(X, Y)= & -g\left(\left(\nabla_{Z} \varphi\right) X, Y\right)-\mu\left[\left(\nabla_{Z} \eta\right)(X) \eta(Y)\right. \\
& \left.+\eta(X)\left(\nabla_{Z} \eta\right)(Y)\right] .
\end{aligned}
$$

Using 2.3 and 2.4 in 3.1 we get

$$
\begin{aligned}
\left(\nabla_{Z} S\right)(X, Y)= & -g\left(g(\varphi Z, \varphi X) \xi+\eta(X) \varphi^{2} Z, Y\right) \\
& -\mu[g(\varphi Z, X) \eta(Y)+g(\varphi Z, Y) \eta(X)] \\
= & -g(\varphi Z, \varphi X) \eta(Y)-g\left(\varphi^{2} Z, Y\right) \eta(X) \\
& -\mu[g(\varphi Z, X) \eta(Y)+g(\varphi Z, Y) \eta(X)] .
\end{aligned}
$$

Using the second term of 2.2 and the first term of 2.1 in 3.2 we have

$$
\begin{aligned}
\left(\nabla_{Z} S\right)(X, Y)= & -g(X, Z) \eta(Y)-g(Y, Z) \eta(X)-2 \eta(X) \eta(Y) \eta(Z) \\
& -\mu[g(\varphi Z, X) \eta(Y)+g(\varphi Z, Y) \eta(X)] .
\end{aligned}
$$

In view of 3.3 it follows that

$$
\begin{aligned}
\left(\nabla_{Z} S\right)(X, Y) & -\left(\nabla_{X} S\right)(Y, Z) \\
= & -g(X, Z) \eta(Y)-g(Y, Z) \eta(X)-2 \eta(X) \eta(Y) \eta(Z) \\
& -\mu[g(\varphi Z, X) \eta(Y)+g(\varphi Z, Y) \eta(X)] \\
& +g(Z, X) \eta(Y)+g(X, Y) \eta(Z)+2 \eta(X) \eta(Y) \eta(Z) \\
& +\mu[g(\varphi X, Z) \eta(Y)+g(\varphi X, Y) \eta(Z)] \\
= & -g(Y, Z) \eta(X)-\mu g(\varphi Z, Y) \eta(X) \\
& +g(X, Y) \eta(Z)+\mu g(\varphi X, Y) \eta(Z) .
\end{aligned}
$$

Since, by hypothesis, the Ricci tensor is of Codazzi type, from (3.4) we get

$$
-g(Y, Z) \eta(X)-\mu g(\varphi Z, Y) \eta(X)+g(X, Y) \eta(Z)+\mu g(\varphi X, Y) \eta(Z)=0 .
$$

Replacing $X$ by $\varphi X$ in 3.5 yields

$$
g(\varphi X, Y) \eta(Z)+\mu g\left(\varphi^{2} X, Y\right) \eta(Z)=0 .
$$

Using the first term of (2.1) in (3.6) we have

$$
g(\varphi X, Y) \eta(Z)+\mu[g(X, Y)+\eta(X) \eta(Y)] \eta(Z)=0 .
$$


Putting $Z=\xi$ in 3.7 we obtain

$$
g(\varphi X, Y)+\mu[g(X, Y)+\eta(X) \eta(Y)]=0 .
$$

Taking a frame field and contracting $X$ and $Y$ yields

$$
\mu=-\frac{\psi}{n-1}
$$

where $\psi=\operatorname{trace} \varphi$.

Using Proposition 2.3 and 3.8 we get

$$
\lambda=-\frac{\psi}{n-1}-n+1
$$

From 3.8 it follows that $\mu \neq 0$.

Thus we can state the following:

Theorem 3.1. An LP-Sasakian manifold with constant scalar curvature whose Ricci tensor is of Codazzi type admits a proper $\eta$-Ricci soliton with $\lambda=-\frac{\psi}{n-1}-$ $n+1, \mu=-\frac{\psi}{n-1}$.

From the above theorem we get:

Corollary 3.2. An LP-Sasakian manifold with constant scalar curvature whose Ricci tensor is of Codazzi type does not admit Ricci solitons with potential vector field $\xi$.

\section{4. $\eta$-RICCI SOLITONS ON $\varphi$-CONFORMALLY SEMI-SYMMETRIC LP-SASAKIAN MANIFOLDS WITH CONSTANT SCALAR CURVATURE}

This section is devoted to the study of $\varphi$-conformally semi-symmetric $\eta$-Ricci solitons on LP-Sasakian manifolds. Then

$$
C \cdot \varphi=0
$$

from which it follows that

$$
C(X, Y) \varphi Z-\varphi(C(X, Y) Z)=0 .
$$

Putting $Z=\xi$ in 4.2 , we get

$$
\varphi(C(X, Y) \xi)=0
$$

Putting $Z=\xi$ in (2.7) and using (2.5), 2.6 and 2.8 we get

$$
\begin{aligned}
C(X, Y) \xi= & {\left[\frac{r}{(n-1)(n-2)}-\frac{1}{n-2}\right]\{\eta(Y) X-\eta(X) Y\} } \\
& -\frac{1}{n-2}[\eta(Y)\{-\varphi X-\lambda X\}-\eta(X)\{-\varphi Y-\lambda Y\}] .
\end{aligned}
$$


In view of 4.3 and 4.4 we have

$$
\begin{aligned}
\varphi(C(X, Y) \xi)= & {\left[\frac{r}{(n-1)(n-2)}-\frac{1}{n-2}\right]\{\eta(Y) \varphi X-\eta(X) \varphi Y\} } \\
& -\frac{1}{n-2}\left[\eta(Y)\left\{-\varphi^{2} X-\lambda \varphi X\right\}-\eta(X)\left\{-\varphi^{2} Y-\lambda \varphi Y\right\}\right] \\
= & 0 .
\end{aligned}
$$

Replacing $X$ by $\varphi X$ in 4.5 we get

$$
\left[\frac{r}{(n-1)(n-2)}-\frac{1}{n-2}\right] \eta(Y) \varphi^{2} X-\frac{1}{n-2}\left[\eta(Y)\left\{-\varphi^{3} X-\lambda \varphi^{2} X\right\}\right]=0,
$$

which implies that

$$
\left[\frac{r}{(n-1)(n-2)}-\frac{1}{n-2}\right]\{X+\eta(X) \xi\}-\frac{1}{n-2}[-\varphi X-\lambda X-\lambda \eta(X) \xi]=0 .
$$

Again replacing $X$ by $\varphi X$ in 4.7 we have

$$
\left[\frac{r}{(n-1)(n-2)}-\frac{1}{n-2}\right] \varphi X-\frac{1}{n-2}\left[-\varphi^{2} X-\lambda \varphi X\right]=0 .
$$

From 4.8 it follows that

$$
\left[\frac{r}{(n-1)(n-2)}-\frac{1}{n-2}+\frac{\lambda}{n-2}\right] \varphi X+\frac{1}{n-2}[X+\eta(X) \xi]=0 .
$$

Taking inner product of 4.9 with respect to $W$ yields

$$
\left[\frac{r}{(n-1)(n-2)}-\frac{1}{n-2}+\frac{\lambda}{n-2}\right] g(\varphi X, W)+\frac{1}{n-2}[g(X, W)+\eta(X) \eta(W)]=0 .
$$

Taking a frame field and contracting $X$ and $W$ in 4.10 yields

$$
\left[\frac{r}{(n-1)(n-2)}-\frac{1}{n-2}+\frac{\lambda}{n-2}\right] \psi+\frac{n-1}{n-2}=0,
$$

where $\psi=\operatorname{trace} \varphi$.

From 4.11 we infer

$$
\lambda=-\frac{n-1}{\psi}-\frac{r}{n-1}+1
$$

Using Proposition 2.3 and 4.12 we get

$$
\mu=-\frac{n-1}{\psi}-\frac{r}{n-1}+n
$$

In view of the above results we can state the following:

Theorem 4.1. A $\varphi$-conformally semi-symmetric LP-Sasakian manifold with constant scalar curvature admits $\eta$-Ricci solitons with $\lambda=-\frac{n-1}{\psi}-\frac{r}{n-1}+1$ and $\mu=-\frac{n-1}{\psi}-\frac{r}{n-1}+n$.

As a corollary of this theorem we have: 
Corollary 4.2. On an LP-Sasakian manifold satisfying $C \cdot \varphi=0$, there is no Ricci soliton with the potential vector field $\xi$.

5. $\eta$-RICCI SOLITONS ON $\varphi$-RICCI SYMMETRIC LP-SASAKIAN MANIFOLDS WITH CONSTANT SCALAR CURVATURE

An LP-Sasakian manifold is said to be $\varphi$-Ricci symmetric if

$$
\varphi^{2}\left(\nabla_{X} Q\right) Y=0
$$

holds for all smooth vector fields $X, Y$. It should be mentioned that $\varphi$-Ricci symmetric Sasakian manifolds have been studied in [13].

With the help of 2.8 we have

$$
Q Y=-\varphi Y-\lambda Y-\mu \eta(Y) \xi .
$$

Taking covariant derivative of $(5.2)$ with respect to an arbitrary vector field $X$ and using $(2.3)$, we get

$$
\begin{aligned}
\left(\nabla_{X} Q\right) Y= & \nabla_{X} Q Y-Q\left(\nabla_{X} Y\right) \\
= & \nabla_{X}[-\varphi Y-\lambda Y-\mu \eta(Y) \xi]+\varphi\left(\nabla_{X} Y\right)+\lambda\left(\nabla_{X} Y\right)+\mu \eta\left(\nabla_{X} Y\right) \xi \\
= & -\varphi\left(\nabla_{X} Y\right)-\left(\nabla_{X} \varphi\right) Y-\lambda\left(\nabla_{X} Y\right)-\mu\left[\nabla_{X} \eta(Y) \xi+\eta(Y) \nabla_{X} \xi\right] \\
& +\varphi\left(\nabla_{X} Y\right)+\lambda\left(\nabla_{X} Y\right)+\mu \eta\left(\nabla_{X} Y\right) \xi \\
= & -\left(\nabla_{X} \varphi\right) Y-\mu\left[\nabla_{X} \eta(Y)-\eta\left(\nabla_{X} Y\right)\right] \xi-\mu \eta(Y) \nabla_{X} \xi \\
= & -g(\varphi X, \varphi Y) \xi-\eta(Y) \varphi^{2} X-\mu\left(\nabla_{X} \eta\right)(Y) \xi-\mu \eta(Y) \varphi X \\
= & -g(X, Y) \xi-2 \eta(X) \eta(Y) \xi-\eta(Y) X-\mu g(\varphi X, Y) \xi-\mu \eta(Y) \varphi X .
\end{aligned}
$$

Operating $\varphi^{2}$ on both sides of 5.3 , we get

$$
\varphi^{2}\left(\nabla_{X} Q\right) Y=-\eta(Y) \varphi^{2} X-\mu \eta(Y) \varphi^{3} X .
$$

Using the first term of (2.1) in (5.4) we obtain

$$
\varphi^{2}\left(\nabla_{X} Q\right) Y=-\eta(Y) X-\eta(X) \eta(Y) \xi-\mu \eta(Y) \varphi X .
$$

In view of (5.1), from 5.5 it follows that

$$
X+\eta(X) \xi+\mu \varphi X=0 .
$$

Replacing $X$ by $\varphi X$ in $(5.6)$ we obtain

$$
\varphi X+\mu[X+\eta(X) \xi]=0
$$

Taking inner product of 5.7 with respect to $W$ we have

$$
g(\varphi X, W)+\mu[g(X, W)+\eta(X) \eta(W)]=0 .
$$

Taking a frame field and contracting $X$ and $W$ in (5.8) yields

$$
\mu=-\frac{\psi}{n-1}
$$

where $\psi=\operatorname{trace} \varphi$.

From 5.9 it follows that $\mu \neq 0$. 
Using Proposition 2.3 and $(5.9)$ we get

$$
\lambda=-\frac{\psi}{n-1}-n+1
$$

Thus we are in a position to state the following:

Theorem 5.1. A $\varphi$-Ricci symmetric LP-Sasakian manifold with constant scalar curvature admits a proper $\eta$-Ricci soliton with $\lambda=-\frac{\psi}{n-1}-n+1, \mu=-\frac{\psi}{n-1}$.

From the above theorem we have the following:

Corollary 5.2. On a $\varphi$-Ricci symmetric LP-Sasakian manifold with constant scalar curvature, there is no Ricci solitons with potential vector field $\xi$.

6. $\eta$-RICCI SOLITONS ON CONFORMALLY RICCI SEMI-SYMMETRIC LP-SASAKIAN MANIFOLDS

In this section we study $\eta$-Ricci solitons on conformally Ricci semi-symmetric LP-Sasakian manifolds, that is,

$$
C \cdot S=0
$$

which implies

$$
(C(X, Y) \cdot S)(Z, W)=0
$$

From 6.2, we get

$$
S(C(X, Y) Z, W)+S(Z, C(X, Y) W)=0 .
$$

Using (2.8) and by the symmetric property of $\varphi$, from (6.3) we get

$$
\begin{aligned}
& -g(C(X, Y) Z, \varphi W)-\lambda g(C(X . Y) Z, W) \\
& \quad-\mu \eta(C(X, Y) Z) \eta(W)-g(\varphi Z, C(X, Y) W) \\
& \quad-\lambda g(C(X, Y) W, Z)-\mu \eta(C(X, Y) W) \eta(Z)=0,
\end{aligned}
$$

which implies that

$$
\begin{aligned}
g(C(X, Y) Z, \varphi W)+\mu \eta( & C(X, Y) Z) \eta(W) \\
& +g(\varphi Z, C(X, Y) W)+\mu \eta(C(X, Y) W) \eta(Z)=0 .
\end{aligned}
$$

Putting $X=W=\xi$ in 6.4 we obtain

$$
g(\varphi Z, C(\xi, Y) \xi)-\mu \eta(C(\xi, Y) Z)+\mu \eta(C(\xi, Y) \xi) \eta(Z)=0 .
$$

With the help of 4.4 we find

$$
\begin{aligned}
\eta(C(\xi, Y) Z)= & g(C(\xi, Y) Z, \xi) \\
=- & g(C(\xi, Y) \xi, Z) \\
=- & {\left[\frac{r}{(n-1)(n-2)}-\frac{1}{n-2}+\frac{\lambda}{n-2}\right] } \\
& \times\{\eta(Y) \eta(Z)+g(Y, Z)\}-\frac{1}{n-2} g(\varphi Y, Z) .
\end{aligned}
$$


Also from 6.6 we get

$$
\eta(C(\xi, Y) \xi)=0 .
$$

Using 6.6 and 6.7 in 6.5 , it follows that

$$
\begin{aligned}
\left\{\frac{r}{(n-1)(n-2)}-\frac{1}{n-2}+\frac{\lambda}{n-2}\right\} g(\varphi Z, \eta(Y) \xi+Y) \\
\quad+\frac{1}{n-2} g(\varphi Z, \varphi Y) \\
\quad+\mu\left\{\frac{r}{(n-1)(n-2)}-\frac{1}{n-2}+\frac{\lambda}{n-2}\right\}\{\eta(Y) \eta(Z)+g(Y, Z)\} \\
\quad+\frac{\mu}{n-2} g(\varphi Y, Z)=0,
\end{aligned}
$$

from which it follows that

$$
\begin{aligned}
\left\{\frac{r}{(n-1)(n-2)}-\frac{1}{n-2}+\frac{\lambda}{n-2}+\frac{\mu}{n-2}\right\} g(\varphi Y, Z) & \\
+\left\{\frac{\mu r}{(n-1)(n-2)}-\frac{\mu}{n-2}\right. & \left.+\frac{\mu \lambda}{n-2}+\frac{1}{n-2}\right\} \\
& \times\{g(Y, Z)+\eta(Y) \eta(Z)\}=0 .
\end{aligned}
$$

Using 2.8 in 6.8 yields

$$
\begin{aligned}
& {\left[\frac{r}{(n-1)(n-2)}-\frac{1}{n-2}+\frac{\lambda}{n-2}+\frac{\mu}{n-2}\right]\{-S(Y, Z)-\lambda g(Y, Z)-\mu \eta(Y) \eta(Z)\}} \\
& +\left[\frac{\mu r}{(n-1)(n-2)}-\frac{\mu}{n-2}+\frac{\mu \lambda}{n-2} \frac{1}{n-2}\right] \times\{g(Y, Z)+\eta(Y) \eta(Z)\}=0
\end{aligned}
$$

From 6.9 we obtain

$$
\begin{aligned}
{\left[\frac{r}{n-1}+(\lambda+\mu-1)\right] S(Y, Z)=(r+\lambda-n \lambda-\lambda \mu+n) } & g(Y, Z) \\
& +\left(1-\mu^{2}\right) \eta(Y) \eta(Z)
\end{aligned}
$$

from which it follows that

$$
S(Y, Z)=a g(Y, Z)+b \eta(Y) \eta(Z)
$$

where

$$
a=\frac{(n-1)(r+\lambda-n \lambda-\lambda \mu+n)}{r+(n-1)(\lambda+\mu-1)} \quad \text { and } \quad b=\frac{(n-1)\left(1-\mu^{2}\right)}{r+(n-1)(\lambda+\mu-1)} .
$$

Theorem 6.1. If a conformally Ricci semi-symmetric LP-Sasakian manifold admits an $\eta$-Ricci soliton, then the manifold is an $\eta$-Einstein manifold, provided $r \neq-(n-1)(\lambda+\mu-1)$. 


\section{EXAMPLE}

We consider a 3-dimensional Lorentzian manifold $M=\left\{(x, y, z) \in \mathbb{R}^{3}\right\}$, where $(x, y, z)$ are the standard coordinates of $\mathbb{R}^{3}$. Let $\left\{e_{1}, e_{2}, e_{3}\right\}$ be a linearly independent global frame on $M$ given by

$$
e_{1}=e^{z} \frac{\partial}{\partial x}, \quad e_{2}=e^{z-a x} \frac{\partial}{\partial y}, \quad e_{3}=\frac{\partial}{\partial z}
$$

where $a$ is a non-zero constant.

Let $g$ be the Lorentzian metric defined by

$$
\begin{gathered}
g\left(e_{1}, e_{3}\right)=g\left(e_{1}, e_{2}\right)=g\left(e_{2}, e_{3}\right)=0, \\
g\left(e_{1}, e_{1}\right)=g\left(e_{2}, e_{2}\right)=1, \quad g\left(e_{3}, e_{3}\right)=-1 .
\end{gathered}
$$

Let $\eta$ be the 1 -form defined by

$$
\eta(X)=g\left(X, e_{3}\right)
$$

for $X \in \chi(M)$. Let $\varphi$ be the $(1,1)$ tensor field defined by

$$
\varphi e_{1}=-e_{1}, \quad \varphi e_{2}=-e_{2}, \quad \varphi e_{3}=0 .
$$

Then using the linearity of $\varphi$ and $g$ we have

$$
\eta\left(e_{3}\right)=-1, \quad \varphi^{2} X=X+\eta(X) e_{3},
$$

and

$$
g(\varphi X, \varphi Y)=g(X, Y)+\eta(X) \eta(Y)
$$

for any $X, Y \in \chi(M)$. Thus for $e_{3}=\xi$, the structure $(\varphi, \xi, \eta, g)$ defines a Lorentzian paracontact structure on $M$.

Let $\nabla$ be the Levi-Civita connection with respect to the Lorentzian metric $g$ and let $R$ be the curvature tensor of $g$. Then we have

$$
\left[e_{1}, e_{2}\right]=-a e^{z} e_{2}, \quad\left[e_{1}, e_{3}\right]=-e_{1}, \quad\left[e_{2}, e_{3}\right]=-e_{2} .
$$

Taking $e_{3}=\xi$ and using Koszul's formula for the Lorentzian metric $g$, we have

$$
\begin{gathered}
\nabla_{e_{1}} e_{1}=-e_{3}, \quad \nabla_{e_{1}} e_{2}=0, \quad \nabla_{e_{1}} e_{3}=-e_{1}, \\
\nabla_{e_{2}} e_{1}=a e^{z} e_{2}, \quad \nabla_{e_{2}} e_{2}=-a e^{z} e_{1}-e_{3}, \quad \nabla_{e_{2}} e_{3}=-e_{2}, \\
\nabla_{e_{3}} e_{1}=0, \quad \nabla_{e_{3}} e_{2}=0, \quad \nabla_{e_{3}} e_{3}=0 .
\end{gathered}
$$

From the above expressions, we see that the manifold under consideration satisfies $\eta(\xi)=-1$ and $\left(\nabla_{X} \varphi\right) Y=g(\varphi X, \varphi Y) \xi+\eta(Y) \varphi^{2} X$. Now we verify the above condition for $X=e_{i}$ and $Y=e_{j}$, for $i, j=1,2,3$.

$$
\begin{aligned}
\left(\nabla_{e_{1}} \varphi\right) e_{1} & =\nabla_{e_{1}} \varphi e_{1}-\varphi\left(\nabla_{e_{1}} e_{1}\right) \\
& =-\nabla_{e_{1}} e_{1}+\varphi e_{3} \\
& =e_{3} . \\
g\left(\varphi e_{1}, \varphi e_{1}\right) \xi+\eta\left(e_{1}\right) \varphi^{2} e_{1} & =g\left(e_{1}, e_{1}\right) e_{3}+0 . \varphi^{2} e_{1} \\
& =e_{3} .
\end{aligned}
$$


Therefore $\left(\nabla_{e_{1}} \varphi\right) e_{1}=g\left(\varphi e_{1}, \varphi e_{1}\right) \xi+\eta\left(e_{1}\right) \varphi^{2} e_{1}$.

$$
\begin{aligned}
\left(\nabla_{e_{1}} \varphi\right) e_{3} & =\nabla_{e_{1}} \varphi e_{3}-\varphi\left(\nabla_{e_{1}} e_{3}\right) \\
& =0+\varphi e_{1} \\
& =-e_{1} . \\
g\left(\varphi e_{1}, \varphi e_{3}\right) \xi+\eta\left(e_{3}\right) \varphi^{2} e_{1} & =0 . e_{3}-\varphi^{2} e_{1} \\
& =\varphi e_{1} \\
& =-e_{1} .
\end{aligned}
$$

Therefore $\left(\nabla_{e_{1}} \varphi\right) e_{3}=g\left(\varphi e_{1}, \varphi e_{3}\right) \xi+\eta\left(e_{3}\right) \varphi^{2} e_{1}$.

$$
\begin{aligned}
\left(\nabla_{e_{2}} \varphi\right) e_{3} & =\nabla_{e_{2}} \varphi e_{3}-\varphi\left(\nabla_{e_{2}} e_{3}\right) \\
& =-\varphi\left(\nabla_{e_{2}} e_{3}\right) \\
& =\varphi e_{2} \\
& =-e_{2} . \\
g\left(\varphi e_{2}, \varphi e_{3}\right) \xi & +\eta\left(e_{3}\right) \varphi^{2} e_{2}=0 . e_{3}-e_{2} \\
& =-e_{2} .
\end{aligned}
$$

Hence $\left(\nabla_{e_{2}} \varphi\right) e_{3}=g\left(\varphi e_{2}, \varphi e_{3}\right) \xi+\eta\left(e_{3}\right) \varphi^{2} e_{2}$.

Similarly we can check for the others $e_{i}, e_{j}$. Hence, $M^{3}(\varphi, \xi, \eta, g)$ is an LPSasakian manifold.

Using the above relations, we can easily calculate the nonvanishing components of the curvature tensor $R$ as follows:

$$
\begin{gathered}
R\left(e_{2}, e_{3}\right) e_{3}=-e_{2}, \quad R\left(e_{1}, e_{3}\right) e_{3}=-e_{1}, \quad R\left(e_{1}, e_{1}\right) e_{2}=\left(1-a^{2} e^{2 z}\right) e_{1}, \\
R\left(e_{2}, e_{3}\right) e_{2}=-a e^{z} e_{1}-e_{3}, \quad R\left(e_{1}, e_{3}\right) e_{1}=-e_{3}, \quad R\left(e_{1}, e_{2}\right) e_{1}=-\left(1-a^{2} e^{2 z}\right) e_{2},
\end{gathered}
$$

and the components which can be obtained from these by the symmetry properties. From the above, we can easily calculate the non-vanishing components of the Ricci tensor $S$ as follows:

$$
S\left(e_{1}, e_{1}\right)=-a^{2} e^{2 z}, \quad S\left(e_{2}, e_{2}\right)=-a^{2} e^{2 z}, \quad S\left(e_{3}, e_{3}\right)=-2 .
$$

Again from 2.9 we obtain $S\left(e_{1}, e_{1}\right)=1-\lambda, S\left(e_{2}, e_{2}\right)=1-\lambda, S\left(e_{3}, e_{3}\right)=\lambda-\mu$. Therefore $\lambda=a^{2} e^{2 z}-1$ and $\mu=a^{2} e^{2 z}+1$. The data $(\varphi, \xi, \eta, g)$ defines an $\eta$-Ricci soliton on the LP-Sasakian manifold with non-constant scalar curvature $r=-2\left(a^{2} e^{2 z}+1\right)$.

\section{References}

[1] Batat, W., Brozos-Vázquez, M., García-Río, E. and Gavino-Fernández, S., Ricci solitons on Lorentzian manifolds with large isometry groups, Bull. London Math. Soc. 43 (2011), no. 6, 1219-1227. MR 2861543.

[2] Blaga, A. M., Eta-Ricci solitons on para-Kenmotsu manifolds, Balkan J. Geom. Appl. 20 (2015), no. 1, 1-13. MR 3367062 .

[3] Blaga, A. M., $\eta$-Ricci solitons on Lorentzian para-Sasakian manifolds, Filomat 30 (2016), no. 2, 489-496. MR 3497930 
[4] Boeckx, E., Kowalski, O. and Vanhecke, L., Riemannian manifolds of conullity two, World Scientific Publishing, 1996. MR 1462887

[5] Brozos-Vázquez, M., Calvaruso, G., García-Río, E. and Gavino-Fernández, S., Threedimensional Lorentzian homogeneous Ricci solitons, Israel J. Math. 188 (2012), 385-403. MR 2897737

[6] Călin, C. and Crasmareanu, M., From the Eisenhart problem to Ricci solitons in $f$-Kenmotsu manifolds, Bull. Malays. Math. Sci. Soc. 33 (2010), no. 3, 361-368. MR 2732157.

[7] Cartan, E., Sur une classe remarquable d'espaces de Riemann, Bull. Soc. Math. France 54 (1926), 214-264. MR 1504900.

[8] Chave, T. and Valent, G., Quasi-Einstein metrics and their renormalizability properties, Helv. Phys. Acta 69 (1996), no. 3, 344-347. MR 1440692

[9] Chave, T. and Valent, G., On a class of compact and non-compact quasi-Einstein metrics and their renormalizability properties, Nuclear Phys. B 478 (1996), no. 3, 758-778. MR 1419065

[10] Chern, S. S., What is geometry?, Amer. Math. Monthly 97 (1990), no. 8, 679-686 MR 1072811

[11] Cho, J. T. and Kimura, M., Ricci solitons and real hypersurfaces in a complex space form, Tohoku Math. J. 61 (2009), no. 2, 205-212. MR 2541405.

[12] De, K. and De, U. C., LP-Sasakian manifolds with quasi-conformal curvature tensor, SUT J. Math. 49 (2013), no. 1, 33-46. MR 3135226

[13] De, U. C. and Sarkar, A., On $\varphi$-Ricci symmetric Sasakian manifolds, Proc. Jangjeon Math. Soc. 11 (2008), no. 1, 47-52. MR 2429328

[14] Deshmukh, S., Jacobi-type vector fields on Ricci solitons, Bull. Math. Soc. Sci. Math. Roumanie 55(103) (2012), no. 1, 41-50. MR 2951970

[15] Deshmukh, S., Alodan, H. and Al-Sodais, H., A note on Ricci solitons, Balkan J. Geom. Appl. 16 (2011), no. 1, 48-55. MR 2785715

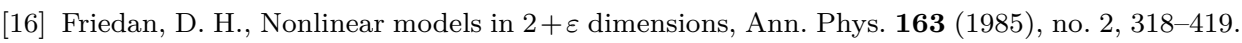
MR 0811072

[17] Gray, A., Einstein-like manifolds which are not Einstein, Geom. Dedicata 7 (1978), no. 3, 259-280. MR 0505561

[18] Hamilton, R. S., The Ricci flow on surfaces, In: Mathematics and general relativity (Santa Cruz, CA, 1986), 237-262. Contemp. Math., 71, Amer. Math. Soc., 1988. MR 0954419.

[19] Ivey, T., Ricci solitons on compact three-manifolds, Differential Geom. Appl. 3 (1993), no. 4, 301-307. MR 1249376

[20] Kowalski, O., An explicit classification of 3-dimensional Riemannian spaces satisfying $R(X, Y) \cdot R=0$, Czechoslovak Math. J. 46(121) (1996), no. 3, 427-474. MR 1408298.

[21] Matsumoto, K., On Lorentzian paracontact manifolds, Bull. Yamagata Univ. Natur. Sci. 12 (1989), no. 2, 151-156. MR 0994289

[22] Matsumoto, K., Mihai, I. and Roşca, R., $\xi$-null geodesic gradient vector fields on a Lorentzian para-Sasakian manifold, J. Korean Math. Soc. 32 (1995), no. 1, 17-31. MR 1321084

[23] Onda, K., Lorentz Ricci solitons on 3-dimensional Lie groups, Geom. Dedicata 147 (2010), 313-322. MR 2660582

[24] Özgür, C., $\varphi$-conformally flat Lorentzian para-Sasakian manifolds, Rad. Mat. 12 (2003), no. 1, 99-106. MR 2022248

[25] Prakasha, D. G. and Hadimani, B. S., $\eta$-Ricci solitons on para-Sasakian manifolds, J. Geom. 108 (2017), no. 2, 383-392. MR 3667227 
[26] Szabó, Z. I., Structure theorems on Riemannian spaces satisfying $R(X, Y) \cdot R=0$. I. The local version, J. Differential Geom. 17 (1982), no. 4, 531-582. MR 0683165

[27] Taleshian, A. and Asghari, N., On LP-Sasakian manifolds satisfying certain conditions on the concircular curvature tensor, Differ. Geom. Dyn. Syst. 12 (2010), 228-232. MR 2606563.

[28] Verstraelen, L., Comments on pseudo-symmetry in the sense of Ryszard Deszcz, In: Geometry and topology of submanifolds, VI (Leuven, 1993/Brussels, 1993), 199-209, World Sci. Publ., 1994. MR 1315102

[29] Yildiz, A. and De, U. C., A classification of $(k, \mu)$-contact metric manifolds, Commun. Korean Math. Soc. 27 (2012), no. 2, 327-339. MR 2962526.

P. Majhi ${ }^{凶}$

Department of Pure Mathematics, University of Calcutta, 35, Ballygaunge Circular Road, Kolkata 700019,

West Bengal, India

mpradipmajhi@gmail.com

D. Kar

Department of Pure Mathematics, University of Calcutta, 35, Ballygaunge Circular Road, Kolkata 700019,

West Bengal, India

debabratakar6@gmail.com

Received: August 17, 2018

Accepted: January 14, 2019 